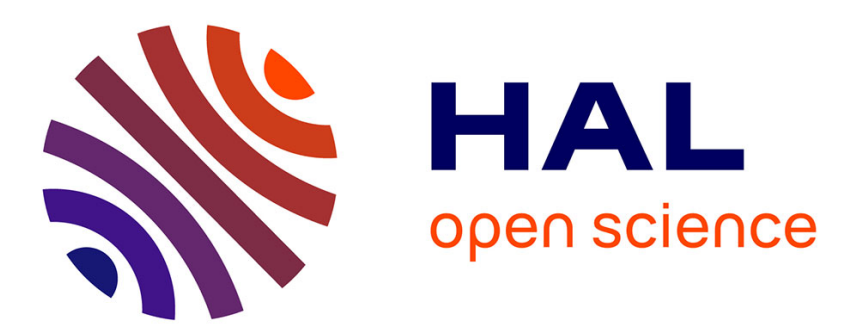

\title{
Subharmonic energy gap structure and gap-enhancement by the Josephson radiation in small dayem bridges
}

P.E. Gregers-Hansen, G.R. Pickett

\section{To cite this version:}

P.E. Gregers-Hansen, G.R. Pickett. Subharmonic energy gap structure and gap-enhancement by the Josephson radiation in small dayem bridges. Revue de Physique Appliquée, 1974, 9 (1), pp.145-152. 10.1051/rphysap:0197400901014500 . jpa-00243724

\section{HAL Id: jpa-00243724 https://hal.science/jpa-00243724}

Submitted on 1 Jan 1974

HAL is a multi-disciplinary open access archive for the deposit and dissemination of scientific research documents, whether they are published or not. The documents may come from teaching and research institutions in France or abroad, or from public or private research centers.
L'archive ouverte pluridisciplinaire HAL, est destinée au dépôt et à la diffusion de documents scientifiques de niveau recherche, publiés ou non, émanant des établissements d'enseignement et de recherche français ou étrangers, des laboratoires publics ou privés. 


\title{
SUBHARMONIC ENERGY GAP STRUCTURE AND GAP-ENHANCEMENT BY THE JOSEPHSON RADIATION IN SMALL DAYEM BRIDGES
}

\author{
P. E. GREGERS-HANSEN and G. R. PICKETT (*) \\ Physics Laboratory I, H C Ørsted Institute \\ University of Copenhagen, Denmark
}

\begin{abstract}
Résumé. - Nous avons observé pour des tensions égales à $2 \Delta / \mathrm{Ne}$ une structure dans la caractéristique $I-V$ de microponts en indium et en étain. Sous l'action d'un rayonnement hyperfréquence ces sous-harmoniques induisent des bandes latérales aux fréquences $(2 \Delta \pm M \hbar \omega) / N e$. Nous expliquons cet effet par la rupture des paires provoquée par le rayonnement Josephson dans la région du micropont et nous l'avons simulé sur un calculateur analogique. La position des sousharmoniques nous permet de déduire que l'énergie du "gap » est supérieure à celle donnée par la théorie BCS et que cet effet est vraisemblablement dû au rayonnement Josephson.
\end{abstract}

\begin{abstract}
We have observed subharmonic energy gap structure in the $I-V$ characteristics of thin film indium and tin microbridges occurring at voltages of $2 \Delta / \mathrm{Ne}$. Under the influence of microwaves the subharmonics develop sidebands at $(2 \Delta \pm M \hbar \omega) / N \mathrm{e}$. We explain the observed behaviour in terms of pair-breaking in the region of the bridge by the Josephson radiation and have simulated the effect by analogue computation. Through the positions of the subharmonic peaks we deduce that the energy near the bridge is enhanced above the BCS bulk value, apparently by the Josephson radiation.
\end{abstract}

1. Introduction. - A number of superconducting junctions is now known which exhibit the Josephson effect. In most of these structures the observed phenomena are fairly well understood. However, thin film bridges (Dayem bridges) first investigated as long ago as 1964 [1] are still only partly understood.

The Dayem bridge consists of a constriction in a thin film of thickness about $0.1 \mu \mathrm{m}$. The width and length of the constriction for various configurations vary a great deal, but for type I superconductors they are normally of the order of the coherence length $\xi$. To observe a behaviour consistent with a sinusoidal current-phase relation, however, we found [2] that the bridges must be smaller than $\xi$. Under these conditions the bridges obeyed surprisingly well the following relationships for current and voltage [3] :

$$
\begin{aligned}
& I=I_{0} \sin \varphi+\frac{V}{R} \\
& V=\frac{\hbar}{2 \mathrm{e}} \frac{\mathrm{d} \varphi}{\mathrm{d} t}
\end{aligned}
$$

where $I_{0}$ is the supercurrent which near $T_{\mathrm{c}}$ was found to be

$$
I_{0}=\frac{\pi}{4 \mathrm{e}} \frac{\Delta(T)^{2}}{k_{\mathrm{B}} T_{\mathrm{c}}} \frac{1}{R}
$$

(*) Permanent address : Department of Physics, University of Lancaster, Lancaster, UK. and where $R$ is the normal resistance of the bridge which sometimes can be observed as a resistance plateau just below $T_{\mathrm{c}}$. These equations lead to an $I-V$ characteristic of the form $V_{\mathrm{DC}}=R \sqrt{I^{2}-I_{0}^{2}}$ and to microwave-induced steps which vary with microwave field as distorted Bessel functions [2]. It is especially the agreement with the experimentally observed size and variation of the steps that has been considered a confirmation of the model. The model also predicts a time variation of the voltage (field current) containing the Josephson frequency and a large number of higher harmonics, the amplitudes of which decrease as a geometric progression [3]

$$
\begin{aligned}
V=V_{\mathrm{DC}}+2 V_{\mathrm{DC}} \sum_{n=1}^{\infty}\left\{\frac{I}{I_{\mathrm{c}}}-\sqrt{\left(\frac{I}{I_{\mathrm{c}}}\right)^{2}-1}\right\}^{n} \times \\
\times \cos \frac{2 n \mathrm{e} V}{\hbar} t
\end{aligned}
$$

and we have been able to observe cavity-induced steps corresponding to the fundamental frequency [4].

The observed behaviour, however, does not always give good agreement with the simple theory. To account for this, one can modify the above model by allowing the current-phase relation to be nonsinusoidal or by adding lumped circuit elements such as inductors and capacitors [5] to the equivalent circuit. Such modifications are however often not very well justified.

We report here new detailed structures in the $I-V$ 
characteristics, appearing at voltages corresponding to $2 \Delta / N$ e where $\Delta$ is the energy gap and $N$ is an integer [6].

These structures in the characteristic may help to improve considerably our understanding of these thin film devices. Our aim here is to describe the observed behaviour in detail and to present an interpretation which is based on pairbreaking.

An interesting consequence of the subharmonic energy gap structure is that we can use it to plot the gap as a function of voltage, or in other words, of Josephson frequency. We find that the gap has a maximum which for a particular voltage exceeds the BCS gap. This seems to indicate the possibility that the gap in a Dayem bridge is enhanced under the influence of the Josephson radiation.

2. The subharmonic structure. - To resolve in greater detail the shape of the $I-V$ characteristic we have measured directly the derivative of voltage with respect to current, $\mathrm{d} V / \mathrm{d} I$. On doing this a regular subharmonic gap structure was always found to be present in our small Dayem bridges. The bridges investigated have been both of indium and tin. The procedure for making them has been described earlier [5] but briefly a narrow cut is made with a razor blade across an evaporated film leaving a microbridge in a narrow perpendicular groove previously etched in the glass substrate. The $I-V$ and $\mathrm{d} V / \mathrm{d} I-V$ curves were measured on two $\mathrm{X}-\mathrm{Y}$ recorders. The current was measured as the voltage drop across a standard resistor, and the voltage $V$ across the bridge was amplified by a Keithley precision nanovolt amplifier. The derivative was measured by the use of a PAR HR8 lock-in amplifier. To avoid the smearing of small peaks in the traces the modulation current from the HR8 was reduced until the shape of the $\mathrm{d} V / \mathrm{d} I-V$ curve was amplitude independent. Negative slopes which often occur in the $I-V$ curves give rise to discontinuities and apparent double peaks in the $\mathrm{d} V / \mathrm{d} I-V$ curves and low slopes cause considerable instability in the derivative, but these cases are readily seen from the $I-V$ curves themselves.

At first the samples were situated in an $X$-band cavity for the study of the influence of microwaves (see below). However, we found that this gave rise to a whole series of self-induced steps corresponding to the many modes in the cavity. These self-induced steps were superposed on the subharmonic structure. All subsequent measurements were made in an open-ended waveguide to eliminate cavity effects.

An example of such a measurement is shown in figure 1 . The $I-V$ curve shown is typical. It deviates considerably from the form $V=R \sqrt{I^{2}-I_{0}^{2}}$ (shown at the top of figure 6). At $2 \Delta$ there is a parallel displacement in the curve and one can just about distinguish a similar smaller displacement at $2 \Delta / 2$. These features, however, are clearly seen when one looks at the $\mathrm{d} V / \mathrm{d} I-V$ curve. There is a large, not very well defined peak in $\mathrm{d} V / \mathrm{d} I$ at $2 \Delta$ which gradually tails off with decreasing voltage. Below this peak there is a whole series of well defined peaks at subharmonics of the energy gap, i. e., at $2 \Delta / N$ e. The peaks at odd $N$ 's in general have a wider linewidth than those at even $N$ 's as can be seen in figure 1 . For small enough bridges, however, this difference becomes negligible.

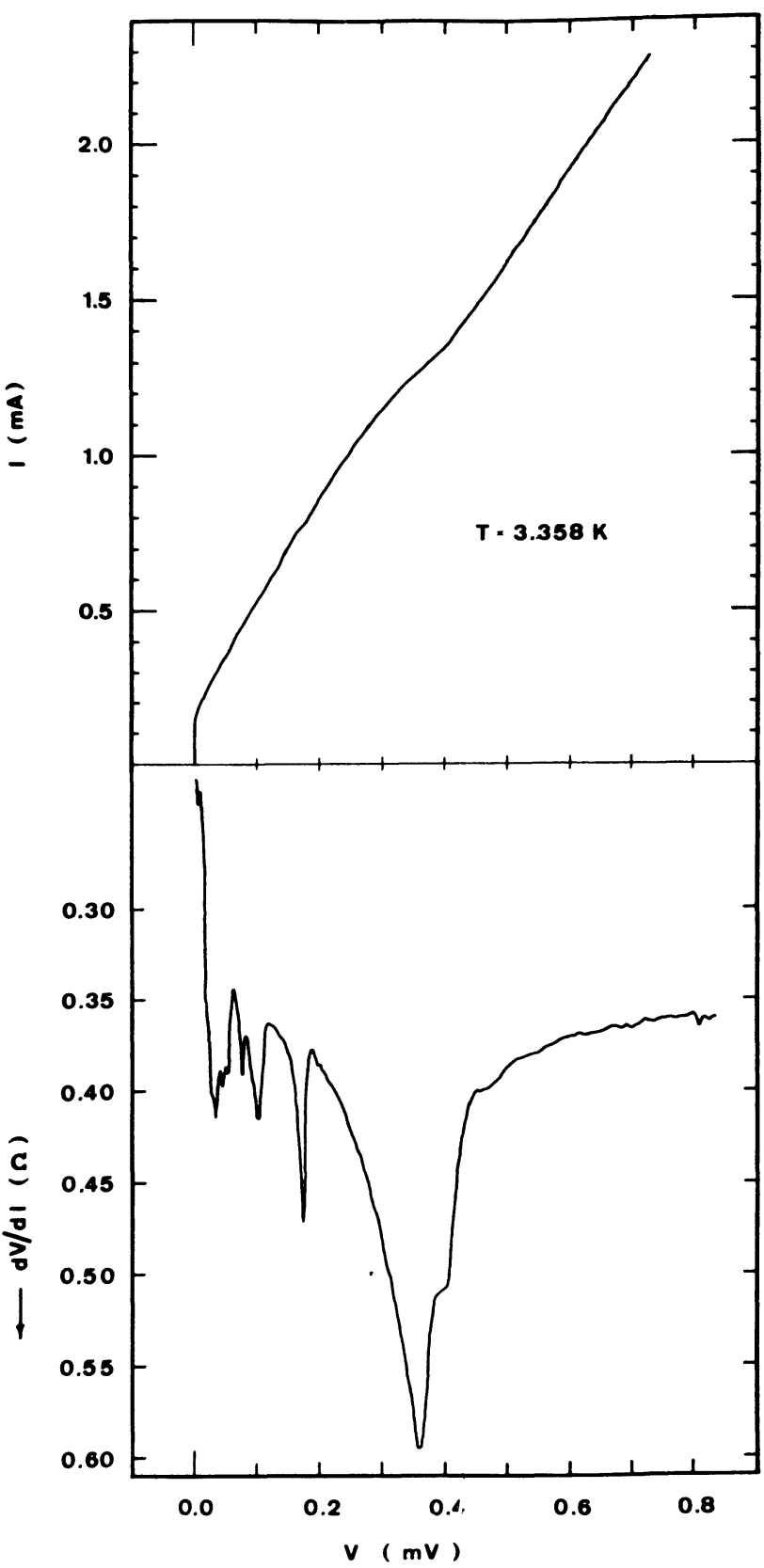

FIG. 1. - Current and $\mathrm{d} V / \mathrm{d} I$ versus voltage for an indium Dayem bridge.

We have investigated the temperature dependence of these peaks and a series of $\mathrm{d} V / \mathrm{d} I-V$ curves are displayed in figure 2 for a series of temperatures. The peak voltages vary with temperature approximately as $\left(1-T / T_{\mathrm{c}}\right)^{1 / 2}$ as expected for the gap near 


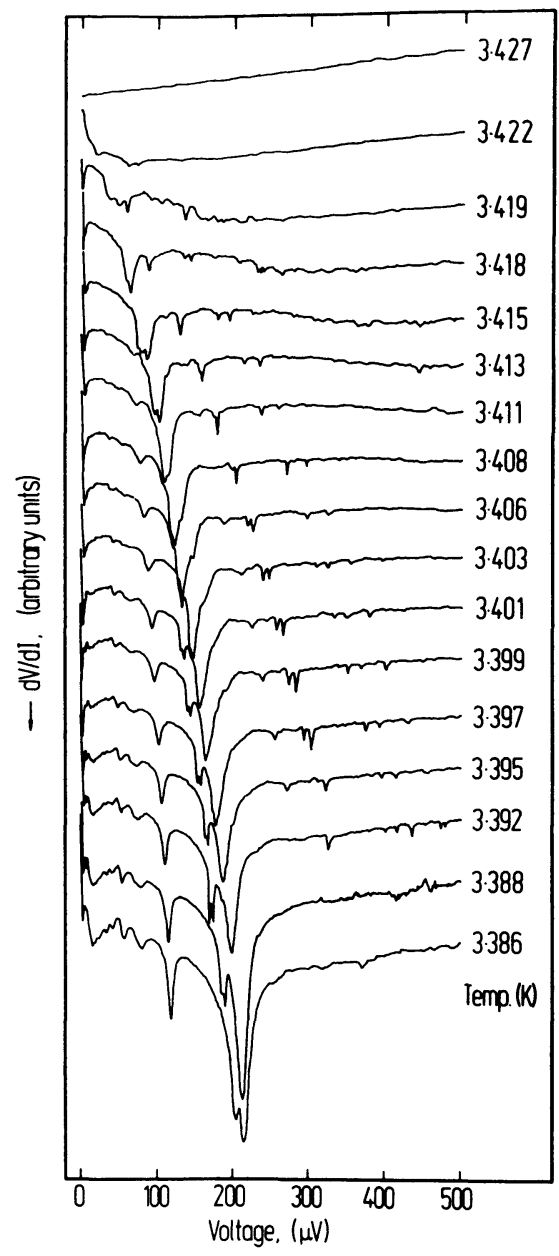

Fig. 2. - Several displaced $\mathrm{d} V / \mathrm{d} I$ vs $V$ curves at different temperatures near the transition temperature. Illustrates the development of the subharmonic gap structure. For the lowest trace $2 \Delta / \mathrm{e}$ is just above $200 \mu \mathrm{V}$. Notice the structure developing above $2 \Delta /$ e.

$T_{\mathrm{c}}$ (compare also with Fig. 8). At voltages higher than $2 \Delta / \mathrm{e}$ there are additional peaks also having the same relative temperature variation as the gap. These may be associated with harmonics of $4 \Delta / \mathrm{e}$, $6 \Delta / \mathrm{e}$, etc. As the temperature is decreased further than shown in figure 2 the subharmonic structure dominates more and more and first the $2 \Delta$-peak and then successively the $2 \Delta / 2 \mathrm{e}, 2 \Delta / 3 \mathrm{e}$, etc., become unstable due to negative or nearly zero slopes in the $I-V$ characteristic at their corresponding voltages. At the same time the relative linewidth of the subharmonic peaks increases with decreasing temperature.

The subharmonic energy gap structure has been observed in a number of small Dayem bridges and all bridges examined show the structure. The size limit where one can observe the regular subharmonic structure has not yet been investigated by scanning electron microscope, but estimates based on previous investigations [7] with similar bridges and investigations by optical microscope tell us that the bridges must have a length and width which is less than about $0.5 \mu \mathrm{m}$. Measurements on larger mask-produced bridges with sizes up to several $\mu \mathrm{m}$ reveal structures which vary with temperature as the energy gap but which have no subharmonic regularity.

3. Influence of a microwave field. - The application of a microwave field has a strong influence on the subharmonic structure. In figure 3 we have displayed the $\mathrm{d} V / \mathrm{d} I$ vs $V$ curves of a relatively small bridge for various values of the relative signal amplitude of a $9 \mathrm{GHz}$ microwave field. As the signal

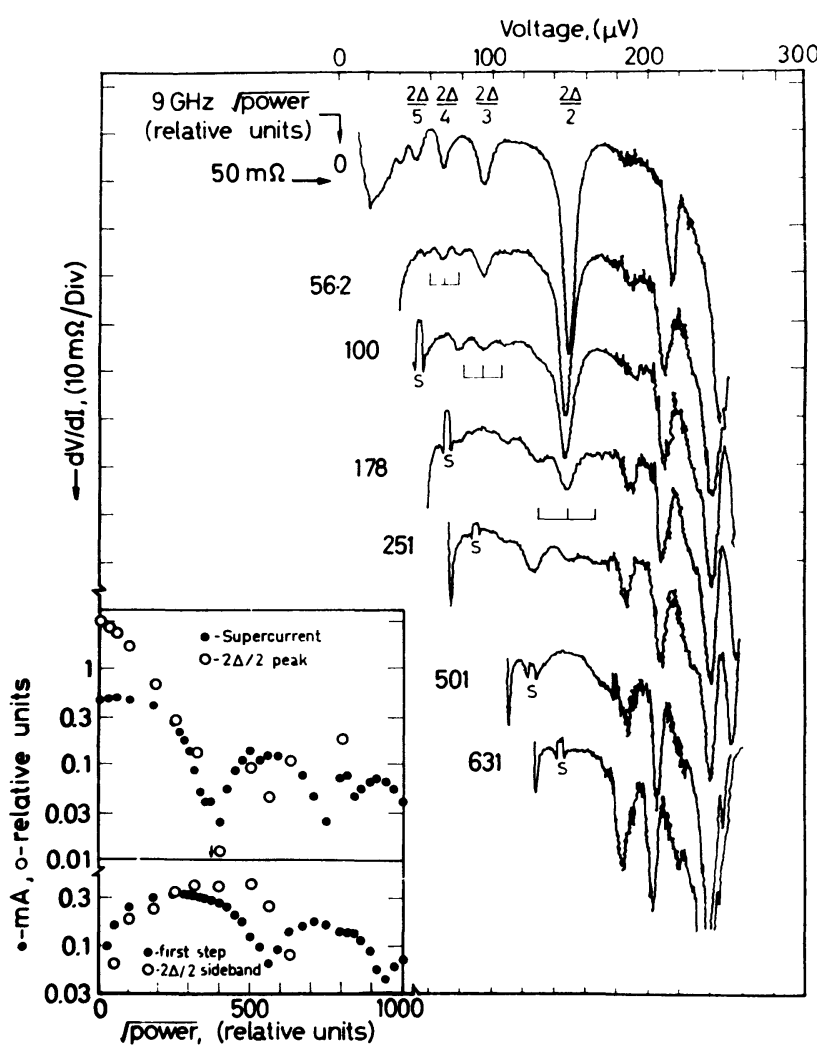

FIG. 3. - Dynamic resistance $\mathrm{d} V / \mathrm{d} I$ versus voltage for various values of relative microwave amplitude. Induced steps are labelled by an $\mathbf{S}$ and the sideband structure is indicated. The height of the peak at $2 \Delta / 2 \mathrm{e}$ and its first sidebands at $2 \Delta / 2 \mathrm{e} \pm \hbar \omega / 2 \mathrm{e}$ are shown in the insert figure as a function of the relative microwave field. Here we have also plotted the supercurrent and the height of the first microwave-induced step versus the same microwave field at the same temperature $T=3.365 \mathrm{~K}$.

amplitude is increased the $2 \Delta / N$ peaks at $N=\ldots 7,6,5$ are rapidly washed out. For the amplitude where the $2 \Delta / 4$ e peak starts to be suppressed one can distinguish two sidebands at $(2 \Delta \pm \hbar \omega) / 4 \mathrm{e}$; but immediately after the Shapiro steps appear on the $I-V$ characteristic and overwhelm the structure. We then observe sidebands at $(2 \Delta \pm \hbar \omega) / 3$ e and follow the reduction of the centre peak to zero and the rise of two sidebands before the whole structure is then obliterated by the steps. The same then happens to the peak at $2 \Delta / 2 \mathrm{e}$ and the sidebands at $(2 \Delta \pm \hbar \omega) / 2 \mathrm{e}$ and we can follow the reduction of the centre peak 
and its reappearance before the structure is overtaken by the Shapiro steps. The estimated heights of the $2 \Delta / 2$ e peak and its two sidebands are plotted as a function of microwave amplitude in the insert. In the same figure we have also plotted for comparison the height of the supercurrent and the first Shapiro step at the same temperature vs the same microwave amplitude. As can be seen the reduction of both the supercurrent and the $2 \Delta / 2$ e peak to zero occurs at the same microwave amplitude and the two sidebands at $(2 \Delta \pm \hbar \omega) / 2$ e vary with microwave amplitude in a way similar to the variation of the first step. At microwave amplitudes beyond that at which the $2 \Delta / 2$ e peak goes to zero the uncertainty in the determination of both the centre peak and its sidebands increases rapidly. From the first part of the plot, however, we can see that the $2 \Delta / 2$ e peak and its sidebands vary in size, respectively, as the square of the supercurrent and the square of the height of the first step. As will be seen later we expect the $2 \Delta / 2$ e peak and its sidebands to vary as Bessel functions squared $I_{0}^{2}\left(2 \mathrm{e} R I_{\mathrm{rf}} / \hbar \omega\right)$ and $I_{1}^{2}\left(2 \mathrm{e} R I_{\mathrm{rf}} / \hbar \omega\right)$ whereas the supercurrent and the first step vary as distorted Bessel functions. The variation of the higher subharmonic peaks is not inconsistent with squared Bessel functions of argument $3 \mathrm{e} R I_{\mathrm{rf}} / \hbar \omega, 4 \mathrm{e} R I_{\mathrm{rf}} / \hbar \omega$, etc. This behaviour is similar to that which is observed for tunnel junctions and point contacts [8]. The broader peak at $2 \Delta$ is also influenced by the microwaves but not in any regular fashion and we do not observe any clearly progressive sidebands.

4. Interpretation. - That the influence of the microwave field is to produce sidebands on the subharmonic structure indicates that the externally applied microwave frequency is mixing with an internal frequency which can only be the ac Josephson oscillations. The peaks in the $\mathrm{d} V / \mathrm{d} I$ curve indicate a depression of the current as the voltage increases through the position of the peak, so we need a mechanism whereby the ac Josephson oscillation depresses the supercurrent at certain voltages. The most obvious possibility is that the Josephson radiation is sufficiently intense to break a considerable number of pairs directly in the bridge region. If we are in a region where the voltage is varying only slowly compared with the coherence length then we would expect direct pair-breaking to be initiated when one of the Josephson harmonics reaches $2 \Delta$, i. e., at $V=2 \Delta / 2 \mathrm{Ne}$. The supercurrent is then depressed by this process at all higher voltages. A further breaking mechanism is introduced as each of the other harmonics reaches $2 \Delta$ and a series of peaks will be observed in the derivative curve.

This is precisely what we see in the case of the even series of peaks. On applying microwaves the Josephson harmonics develop sidebands at $2 N e V \pm M \hbar \omega$ ( $N$ and $M$ being integers) and thus we should expect sidebands to the subharmonic peaks when these sideband frequencies reach $2 \Delta$ i. e., at $V=(2 \Delta \pm M \hbar \omega) / 2 N e$ which is again what we see for the even peaks.

The broader and less well-defined odd series of peaks is not so straightforward to explain. However, the fact that the series is governed by odd numbers provides the clue to the explanation which is in processes where pair-breaking is accompanied by a quasi-particle crossing the bridge in one or the other direction. The quasi-particles resulting from a broken pair appear at a distance from each other of the order of the coherence length.

Referring now to figure 4, we have drawn a diagrammatic bandstructure representation of a bridge in which the voltage varies smoothly from one side

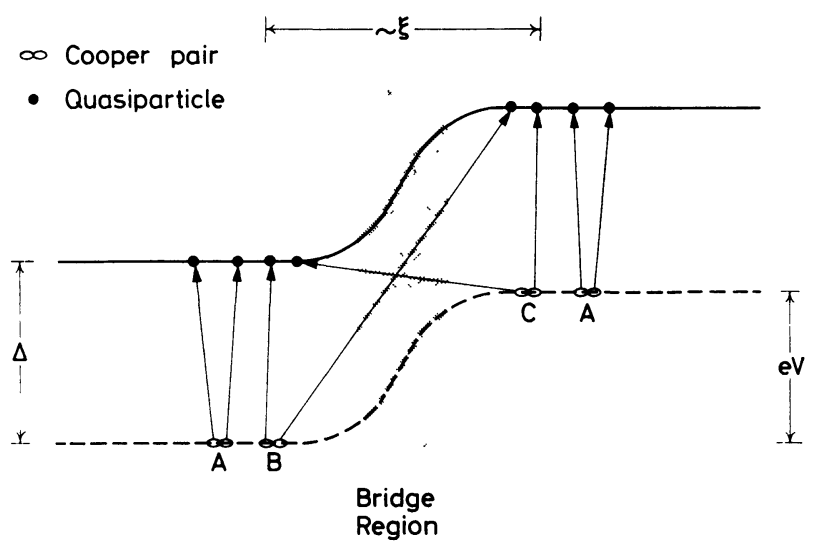

Fig. 4. - Semiconductor model of the variation of the superconducting energy gap through the bridge region when a voltage difference $V$ is applied. The Josephson photon field is thought to cause depairing through the 3 processes $A, B$ and $C$ indicated. Processes labelled A give rise to the even lines in the subharmonic structure. The processes $\mathrm{B}$ and $\mathrm{C}$ where the quasi-particles appear on opposite sides of the bridge require energies of $2 \Delta+\mathrm{e} V$ and $2 \Delta-\mathrm{e} V$, respectively, and give rise to the odd lines. The coherence length $\xi$ has been drawn somewhat longer than the region of voltage drop across the bridge.

to the other. The processes labelled $A$ represent the simple pair-breaking discussed above. Processes labelled $\mathrm{B}$ and $\mathrm{C}$ represent pair-breakings where the two quasi-particles appear on opposite sides of the bridge. Assuming that both particles appear at the density of states singularities at the bottom of the quasi-particle band then the limiting energies for these processes are $2 \Delta-\mathrm{e} V$ and $2 \Delta+\mathrm{e} V$, and the corresponding subharmonic peaks appear at voltages of $2 \Delta /(2 N+1)$ e and $2 \Delta /(2 N-1)$ e which gives us the odd series of lines. On irradiation with microwave sidebands should appear at

$$
(2 \Delta \pm M \hbar \omega) /(2 N \pm 1) \text {. }
$$

It is interesting to notice that the processes $B$ and $\mathrm{C}$ have their counterpart in tunnel junctions where they give rise to the so-called quasi-particlepair interference term [9].

On the basis of this explanation the mixing of the 
external frequency and the Josephson radiation which governs the subharmonic sideband structure is similar to that which leads to the microwave induced steps in the $I-V$ characteristic. In the case of the steps the mixing is between the external microwaves and all the harmonics of the Josephson frequency, whereas for the sidebands to the subharmonic peaks only one of the harmonics is mixed with the external microwaves. However, if the Josephson oscillation contains only small amounts of higher harmonics (i. e., $\hbar \omega / 2 \mathrm{e} R I_{0}$ of the order of or bigger than 1) we would expect that the two corresponding phenomena relating to the fundamental Josephson frequency, i. e., $2 \Delta / 2$ e peak and its first sidebands and the supercurrent and first microwave-induced step should have the same power dependence. This is rather well confirmed in the inset of figure 3. However, the pair-breaking rate is an intensity effect so we would expect the subharmonic structure to follow Bessel functions squared, as we observe.

Figure 4 has been drawn on the assumption that the bridge length is comparable to the coherence length. However, if the bridge is very short compared to $\xi$ then the probabilities of the three processes $\mathrm{A}, \mathrm{B}$ and $C$ are much higher than those of any intermediate processes and both odd and even lines should be of sharpness comparable to that of the singularity in the quasi-particle density of states. However, if we increase the bridge length to be comparable with the coherence length, process $A$ will still be expected to give a relatively sharp peak whereas $B$ and $C$ processes coming on the sloping part of the voltage curve will become rather broadened.

The relative intensity of the even to the odd series of lines is an indication of the distance into the bulk film that the Josephson radiation can pair-break. In all the bridges tested the even series is of comparable or somewhat lower intensity than the odd series which suggests that the pair-breaking only extends a distance of the order of the coherence length from the bridge region.

At temperatures close to $T_{\mathrm{c}}$ the coherence length is so large that the linewidths are not influenced by it. However, as the temperature is reduced the decreasing coherence length becomes at some point comparable with the length of the bridge and the linewidths should begin to increase. In figure 5 we have plotted the relative linewidth of the $2 \Delta / 2$ e, $2 \Delta / 3$ e and $2 \Delta / 4$ e peaks of the same sample as that of figure 3 as a function of the gap calculated from the position of the subharmonic peaks, and the increase in width when the coherence length becomes comparable to the size of the bridge is quite clear. The change in slope occurs at a temperature of $3.2 \mathrm{~K}$. Taking the zero temperature coherence length as $1200 \AA$ would give a coherence length $\xi=\xi_{0}\left(1-T / T_{\mathrm{c}}\right)^{-1 / 2}$ of about $3000 \AA$ at the break in slope which is about the bridge dimension that we estimate. As seen in figure 5 the linewidth of the even and the odd series starts to increase at approximately the same gapvalue (temperature). This again indicates that the pair-breaking is localized within a coherence length from the bridge.

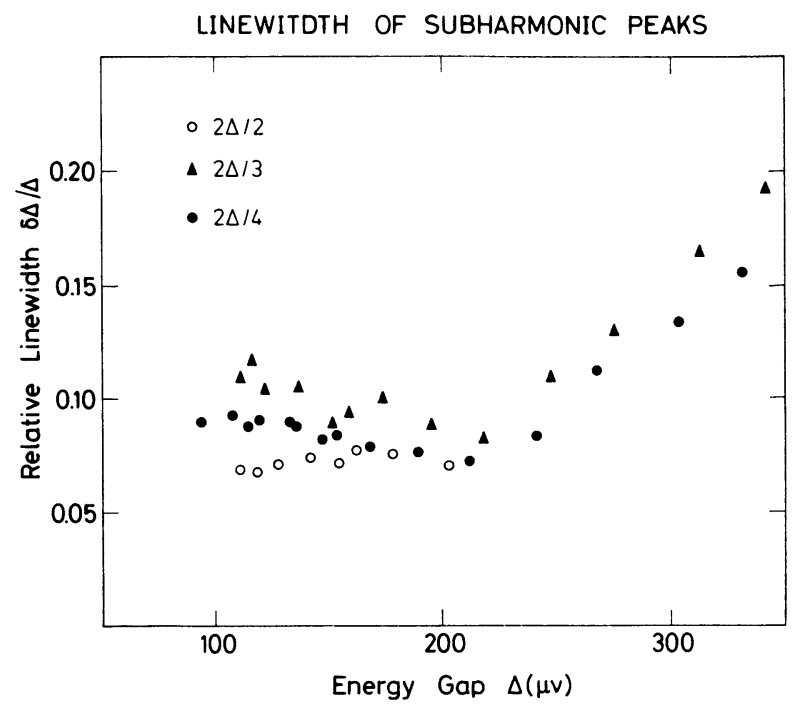

FIG. 5. - The width of the subharmonic peaks measured at half their maximum height plotted against the energy gap determined as $N / 2$ times the position of the $2 \Delta / N$ e peak. The linewidth of the $2 \Delta / 2 \mathrm{e}, 2 \Delta / 3 \mathrm{e}$ and $2 \Delta / 4 \mathrm{e}$ peaks are shown in the temperature region where they could be studied reliably. The lowest temperature available is where the $I-V$ characteristic gets negative resistance regions. The scatter in the points stems from difficulties in measuring the linewidth from our $\mathrm{d} V / \mathrm{d} I$ vs $V$ curves which get increasingly difficult at low gap values (voltages). The break in the slope where $\xi$ becomes of the order of the bridge length occurs at $3.2 \mathrm{~K}$.

This semiconductor type of approach assumed in figure 4 is of course an oversimplification as the assumption of a constant gap across the bridge is certainly suspect. However, the behaviour of the gap in the region of rapidly changing voltage does not qualitatively change the above discussion.

5. Analogue computation. - In order to study which frequencies are available for pair-breaking in small Dayem bridges we have performed an analogue simulation using the two-fluid model in the same way as first performed by Russer [10]. The equivalent circuit used is shown in figure 6. The normal electron current flow across the bridge is governed by the normal resistance $R$ which is in parallel with the supercurrent determined by the Josephson equations. The circuit is driven by a dc current source across which a " microwave " induced current $I_{1} \sin \omega t$ is applied. A frequency analyzer set at a single frequency (simulating the gap frequency) detects the amplitude of the radiation in the bridge analogue. The adjustable parameters ; $R$, the normal resistance; $I_{0}$, the supercurrent; the integration time constant of the computer and the analyzer frequency and bandwidth are adjusted to reproduce as nearly as possible the conditions under which the 
experimental curves of figure 3 were taken. Setting the analyzer to a single frequency equivalent to $2 \Delta$ means that we are only observing frequencies that would give us the even lines in the subharmonic structure.

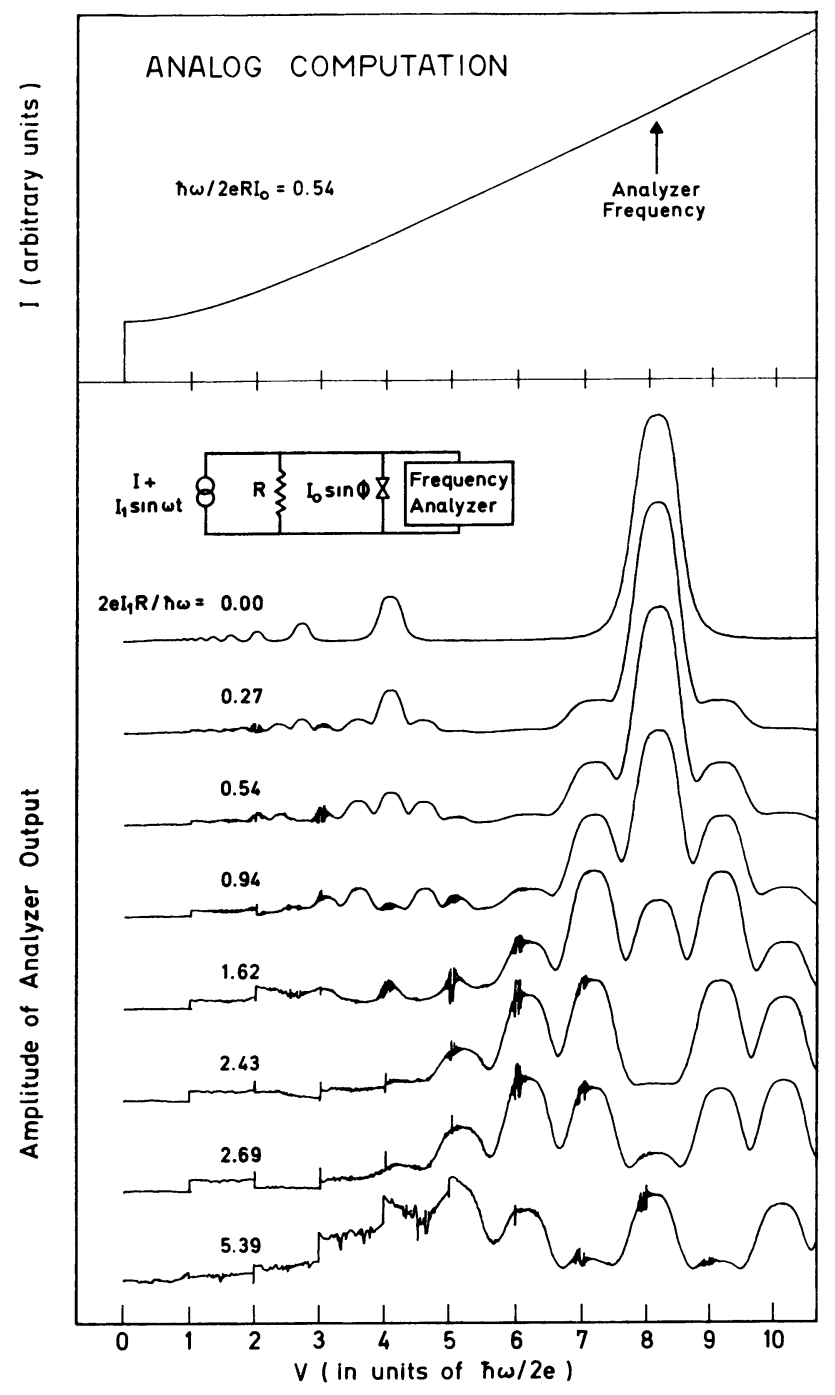

FIG. 6. - Analogue simulation of the amplitude of the pairbreaking frequency which in turn gives rise to the subharmonic energy gap structure. Upper figure shows the $I-V$ characteristic in scaled units; the arrow indicates the voltage where the fundamental Josephson frequency hits the analyzer frequency. The equivalent circuit forming the basis of the analogue simulation is shown just below this. The frequency window has a relative width of about $10 \%$. The lower traces show the analyzer output versus scaled voltage for various values of an applied AC signal amplitude simulating the microwaves. This figure is to be compared to the experiments shown in figure 3 .

The result of the simulation is shown in figure 6 . The top part of the diagram shows the simple $I-V$ characteristic. The lower curves show the output of the frequency analyzer as a function of voltage for a series of microwave amplitudes. A comparison with the behaviour of the even harmonics in the experimental curves of figure 3 reveals remarkable agreement. With zero microwave power the analogue curve shows a large peak due to the fundamental Josephson frequency with the subharmonics falling off in regular progression. On applying an ac amplitude of $2 \mathrm{e}_{1} R / \hbar \omega=0.27$ the third peak from the right has well developed sidebands but has already been overtaken by the microwave-induced steps. The steps in the characteristic are visible because of the analyzer frequency bandwidth used. The second peak has developed sidebands with a step appearing just alongside. This is in close correspondence to the sidebands appearing on the $2 \Delta / 4$ e peak in figure 3 at the relative root power equal to 56.2. The main peak in the computation falls to zero at a microwave amplitude of about 2.43. A step is just developing on the first sideband. This is about equivalent to the root power 501 trace in figure 3. In the root power 631 trace in this figure the main peak is just beginning to increase again as a step appears right on top of it. This is equivalent to the situation in the computation somewhere between amplitudes of 2.69 and 5.39.

The close correspondence between simulation and observation is gratifying and indicates quite clearly that the subharmonic structure is a function of the detection of the Josephson radiation from the bridge by the energy gap.

We have not attempted to analyze at several frequencies to simulate the odd peaks. These are less simple having contributions from two adjacent Josephson harmonics and the power dependences of the peaks and sidebands is thus more complex. In figuer 3 the sidebands of the $2 \Delta / 3$ e peak do in fact have a rather poorly-defined power dependence.

6. Enhancement of the energy gap by the Josephson radiation. - The subharmonic structure has the extremely useful property that it displays the gap in a very direct way making it a very good probe of the superconducting properties of the bridge region itself. We can regard the structure as a spectroscopic analysis of the gap at various values of the voltage or, in other words, at various values of the Josephson frequency. The gap we get directly from the voltage of the peaks at $2 \Delta / N$ e and can plot $\Delta$ as a function of the dc voltage bias. Doing this we find that the gap is not a constant, as one might at first expect, but increases with increasing voltage to a maximum and then falls again, as shown in figure 7 . This behaviour is very similar to the observations by Dayem and Wiegand [11] of the increase in supercurrent $I_{0}$ under the influence of an external microwave field. In figure 8 we have plotted a further series of subharmonics, this time as a function of temperature rather than voltage. The continuous lines represent the corresponding submultiples of the BCS bulk gap. It is quite clear that at any voltage (frequency) there is a maximum and minimum temperature between which there is gap enhancement and at any temperature there is a corresponding 


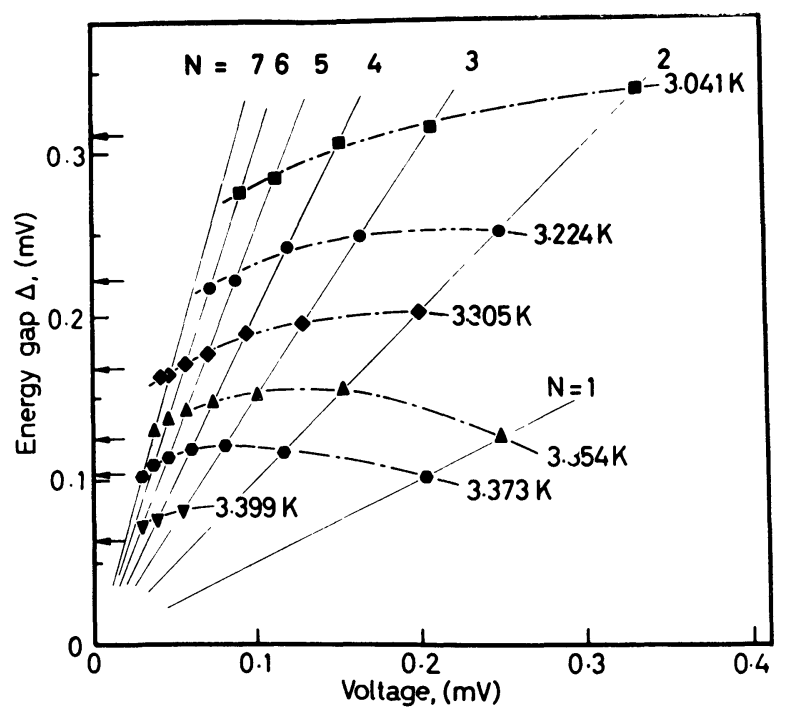

FIG. 7. - The energy gap determined from the voltages of the peaks in the $\mathrm{d} V / \mathrm{d} I$ vs $V$ curve $(\Delta=N \mathrm{e} V / 2)$ plotted against voltage for six different temperatures. The six arrows indicate the indium BCS gap at the same temperatures. Notice the similarity between this figure and figure 10 in reference [11].

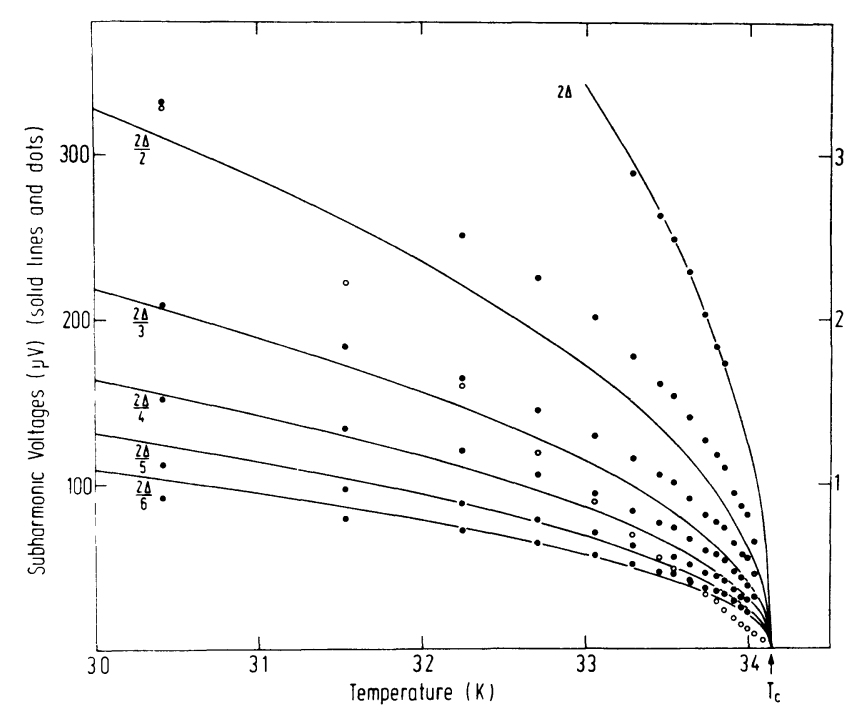

FIG. 8. - Voltage positions of the peaks in $\mathrm{d} V / \mathrm{d} I$ at submultiples of the energy gap plotted as a function of temperature (filled circles) compared to the temperature dependence of submultiples of the BCS energy gap (solid lines). The open circles show the temperature dependence of the supercurrent (linear). The transition temperature of the background film and of the bridge differs only about $3 \mathrm{mK}$. The figures $3,5,7$ and 8 refers to the same sample.

maximum and minimum voltage (frequency), again very similar to the Dayem and Wiegand's (and our own) findings for the enhancement of $I_{0}$.

What we presume we are seeing is the enhancement of the gap in the bridge region under the influence of the strong microwave field generated by the Josephson oscillations. The possibility of the enhancement of the gap in a thin superconducting film by a microwave field has been discussed in several papers by
Eliashberg and co-workers [12] and it is presumably the self-excitation of this effect that we are observing.

We can of course observe directly from the subharmonic structure the effect of an external microwave field on $\Delta$, for instance by examining the shifts in the voltage of the peaks in figure 3 , but in all cases that we have examined external microwaves depress the gap with increasing amplitude. This is not surprising as gap-enhancement by the Josephson oscillations increases $I_{0}$ and thus increases the strength of the selfmicrowave field giving positive feedback, and we would expect the gap to be excited to something like its optimum value for the particular frequency.

At zero voltage where there is no competition from self-enhancement then external microwaves can enhance $\Delta$, and consequently $I_{0}$, as suggested by Dayem and Wiegand [11]. We have tried to observe enhancement by external microwaves very near to $V=0$ but our bridges do not show enough structure in the $\mathrm{d} V / \mathrm{d} I$ curves in this region to allow any effect to be seen.

If the gap increases with increasing voltage due to self-enhancement then we would also expect the supercurrent to increase. Referring back to the simple equivalent circuit used in the analogue computations, we get for a constant $I_{0}$ a concave $I-V$ characteristic as shown in the top part of figure 6. However, the experimental curves all have a tendency to convexity as seen in figure 1, and this could at least to some extent be due to $I_{0}$ increasing with increasing voltage.

The question arises that if we are seeing a gap enhancement by a microwave field why has this effect been conspicuously absent from all thin film configurations apart from constrictions, perhaps most notably from tunnel junctions, where the gap is easily monitored. We feel that to observe this effect extremely high microwave amplitudes may be necessary. The principal feature which characterizes constrictions is that very high microwave currents can be readily generated in the film at the bridge and thus the microwave field acting on the electron gas can be very high indeed. It is difficult to generate fields of equivalent intensity in the relatively extensive films of tunnel junctions. An independent verification could be provided by laying a constricted film as one side of an S-I-S tunnel junction and monitoring any change in $\Delta$ in the constriction induced by microwave excitation through the usual tunneling characteristic.

7. Conclusion. - We have described and interpreted subharmonic energy-gap structure in Dayem bridges on the basis of pair-breaking by the harmonics of the Josephson radiation. Similar structures have been observed several times before in several types of weak link, particularly in tunnel junctions, but we feel, as also concluded by Rowell and Feldmann [13] that the majority of this structure must be due to microbridge-like short circuits of one sort or another in the devices. Depending on whether the observed 
$I-V$ characteristics are above or below that in the normal state a depression of the supercurrent (and gap) at submultiples of the gap would possibly give rise to different types of line-shapes in the $\mathrm{d} V / \mathrm{d} I$ vs $V$ curves.

The subharmonic structure itself gives a particularly clear picture of the gap rather as does a tunneling characteristic and we feel this property may be useful in the study of the dynamic properties of not only constricted films but superconductors in general. We have used the structure to observe the enhancement of the gap by the ac Josephson radiation in the bridge. It is perhaps something of a paradox that the gap-enhancing properties of the radiation are only manifest through its pair-breaking ability.

Acknowledgments. - The authors would like to acknowledge the collaboration of Mogens Levinsen and Elbert Hendricks at various stages of this work and would also like to thank W. T. Band for several helpful discussions. We also acknowledge K. Søe Højbjerg, AEK, Risø for help with the analogue computer and the expert technical assistance of Ole Eg and B. Hermann Pettersson.

\section{References}

[1] Anderson, P. W. and Dayem, A. H., Phys. Rev. Lett. 13 (1964) 195.

[2] Gregers-Hansen, P. E. and Levinsen, M. T., Phys. Rev. Lett. 27 (1971) 848.

[3] Aslamazov, L. G. and Larkin, A. I., Pis'ma Zh. Eksp. Teor. Fiz. 9 (1969) 150 (JETP Lett. 9 (1969) 87).

[4] Levinsen, M. T., Revue Phys. Appl. 9 (1974) 135.

[5] Gregers-Hansen, P. E., Hendricks, E., Levinsen, M. T. and Fog Pedersen, G., Proc. 1972 Appl. S.-C. Conf., IEEE Conf. Rec. NCHO682-5-TABSC, p. 597.

[6] Gregers-Hansen, P. E., Hendricks, E., Levinsen, M. T. and Pickett, G. R., Phys. Rev. Lett. 31 (1973) 524.
[7] Gregers-Hansen, P. E., Levinsen, M. T. and Fog PederSEN, G., J. Low Temp. Phys. 7 (1972) 99.

[8] Hoffmann Sørensen, O., Kofoed, B., Pedersen, N. F. and Shapiro, S., Revue Phys. Appl. 9 (1974) 153.

[9] Langenberg, D. N., Revue Phys. Appl. 9 (1974) 35.

[10] Russer, P., Acta Phys. Austriaca 32 (1970) 373.

[11] Dayem, A. H. and Wiegand, J. J., Phys. Rev. 155 (1967) 419.

[12] Ivlev, B. I., Lisitsyn, S. G. and Eliashberg, G. M., J. Low Temp. Phys. 10 (1973) 449.

[13] Rowell, J. M. and Feldman, W. L., Phys. Rev. 172 (1968) 393. 